\title{
Motor neuron apoptosis and neuromuscular junction perturbation are prominent features in a Drosophila model of Fus-mediated ALS
}

\author{
Ruohan Xia', Yajuan Liu', Liuqing Yang ${ }^{2}$, Jozsef Gal ${ }^{2}$, Haining Zhu ${ }^{2 *}$ and Jianhang Jia ${ }^{1,2^{*}}$
}

\begin{abstract}
Backgound: Amyotrophic lateral sclerosis (ALS) is progressive neurodegenerative disease characterized by the loss of motor function. Several ALS genes have been identified as their mutations can lead to familial ALS, including the recently reported RNA-binding protein fused in sarcoma (Fus). However, it is not clear how mutations of Fus lead to motor neuron degeneration in ALS. In this study, we present a Drosophila model to examine the toxicity of Fus, its Drosophila orthologue Cabeza (Caz), and the ALS-related Fus mutants.

Results: Our results show that the expression of wild-type Fus/Caz or FusR521G induced progressive toxicity in multiple tissues of the transgenic flies in a dose- and age-dependent manner. The expression of Fus, Caz, or FusR521G in motor neurons significantly impaired the locomotive ability of fly larvae and adults. The presynaptic structures in neuromuscular junctions were disrupted and motor neurons in the ventral nerve cord (VNC) were disorganized and underwent apoptosis. Surprisingly, the interruption of Fus nuclear localization by either deleting its nuclear localization sequence (NLS) or adding a nuclear export signal (NES) blocked Fus toxicity. Moreover, we discovered that the loss of caz in Drosophila led to severe growth defects in the eyes and VNCs, caused locomotive disability and NMJ disruption, but did not induce apoptotic cell death.

Conclusions: These data demonstrate that the overexpression of Fus/Caz causes in vivo toxicity by disrupting neuromuscular junctions (NMJs) and inducing apoptosis in motor neurons. In addition, the nuclear localization of Fus is essential for Fus to induce toxicity. Our findings also suggest that Fus overexpression and gene deletion can cause similar degenerative phenotypes but the underlying mechanisms are likely different.
\end{abstract}

Keywords: ALS, Fus, Caz, Locomotion, Neurodegeneration, Drosophila

\section{Background}

Amyotrophic lateral sclerosis (ALS, also known as Lou Gehrig's disease) is a motor neuron disease that causes a disabling condition whereby the degeneration of motor neurons causes progressive muscle weakness and leads to death usually within five years of disease onset [1]. Most cases of ALS occur sporadically, however a small percentage of cases are inherited. Several ALS genes have been identified, and mutations within them can lead to familial ALS [2], including $\mathrm{Cu} / \mathrm{Zn}$ superoxide dismutase (SOD1) [3], TDP-43 [4] and Fused in

\footnotetext{
* Correspondence: haining@uky.edu; jianhang.jia@uky.edu

'Markey Cancer Center, University of Kentucky, KY 40536 Lexington, USA ${ }^{2}$ Department of Molecular and Cellular Biochemistry, College of Medicine, University of Kentucky, KY 40536 Lexington, USA

Full list of author information is available at the end of the article
}

sarcoma (Fus) [5-8]. In addition to familial ALS, Fus mutations are also implicated in sporadic ALS cases [9-11]. Both Fus and TDP-43 are DNA- and RNA-binding proteins. While TDP-43 has been intensively studied, the role of Fus in the ALS etiology is largely unknown.

Fus is a ubiquitous multi-domain RNA-binding protein [12] involved in many processes of RNA metabolism including transcriptional regulation, mRNA splicing and mRNA shuttling between the nucleus and the cytoplasm $[13,14]$. In neurons, Fus is also implicated in the transportation of mRNA for local translation in dendrites $[15,16]$. Fus mainly localizes in the nucleus, but has also been shown to present at lower levels in the cytoplasm of most cell types, including neurons and 
glial cells $[17,18]$. The ALS-related mutations are clustered in the carboxyl-terminus of Fus and exhibit an abnormal nucleo-cytoplasmic redistribution and cytosolic inclusions in the motor neurons in familial ALS patients $[7,8]$. Studies at the cellular level have shown that the nuclear localization sequence (NLS) located in the C-terminus of Fus is necessary and sufficient for nuclear targeting of Fus [19]. ALS mutations within the NLS significantly impair the nuclear targeting function of the sequence, leading to the cytoplasmic accumulation of mutant Fus. Mutations in the NLS have also been shown to promote Fus co-localization with stress granules in the cytosol [19-21]. However, it is still unclear how the mutations cause the toxicity. Moreover, wild-type Fus aggregation has also been reported in several ALS cases. Thus, it is important to determine whether the cytoplasmic or nuclear fraction of Fus is critical to cause the toxicity in ALS. The tentative hypothesis in the field is that cytoplasmic mislocalization and inclusions are important during the disease process. However, this remains to be thoroughly tested.

Appropriately constructed animal models are valuable for defining the link between gene mutations and the ALS disease. Well-established genetics, small genome size, minimal genetic redundancy, and ease of handling have made Drosophila an ideal model system to study conserved mechanisms that are involved in human diseases. Studies using Drosophila as a model have shown that mutating TDP-43 causes locomotive defects [22], whereas overexpressing TDP-43 causes neurotoxicity [23], most likely through genetic interactions with TER94, which is an ATPase associated with multiple cellular activities [24]. These findings suggest that Drosophila is a powerful animal model for ALS. Studies of transgenic Drosophila lines with the overexpression of Fus have been reported in the past [25-27], but the molecular mechanisms underlying Fus/Caz function in Drosophila remain elusive. The Drosophila homologue of Fus, Cabeza (Caz), is enriched in the brain and central nervous system [28,29]. However, the functional link between Drosophila Caz and human Fus has not yet been established.

In this study, we generated and characterized transgenic flies expressing wild-type Fus, several ALS-related Fus mutants, and wild-type Caz using different tissuespecific Gal4 lines. We found that toxicity was dependent on the expression level of Fus/Caz in all tissues examined. Flies overexpressing Fus or Caz by the motor neuron-specific Gal4 showed significantly impaired larval crawling and adult fly movement. Defects in the neuromuscular junctions (NMJs) were evident in these flies. In addition, motor neurons in the ventral nerve cord (VNC) of these flies were disorganized and showed elevated apoptosis. More interestingly, C-terminal NLS is essential for Fus toxicity, since both the deletion of the last 32 amino acids in Fus (Fus $\triangle 32$ ) and the addition of an NES to wild type full-length Fus (FusNES) dramatically reduced toxicity in vivo. Finally, we analyzed the caz mutant flies and found that the loss of Caz induced phenotypes in Drosophila eyes, VNC neurons, and NMJs. The lack of Caz in motor neurons caused similar NMJ defects and locomotive impairment as the Fus/Caz overexpressing flies, but did not cause apoptosis in motor neuron cell bodies. Thus, the mechanisms underlying the toxicity caused by Caz deletion and Fus/Caz overexpression are likely different. The genetic model we generated shows that Fus/Caz is required for neuronal function in vivo and that elevated levels of Fus/Caz in the nucleus can induce neuronal toxicity. The findings of this study provide insights into the molecular mechanisms of Fus/Caz in motor neuron degeneration and ALS

\section{Results}

The expression of Fus/Caz results in wing defects and decreased viability in Drosophila

Animal models for neurodegenerative diseases often involve overexpression of disease relevant proteins in yeast, Drosophila, C. elegans, and mouse. We generated transgenic flies with the PhiC31 integration system to integrate human Fus or Drosophila homolog Caz cDNA at the $75 \mathrm{~B} 1$ attP locus in the Drosophila genome to ensure that the proteins were expressed at the same level without positional effects [30,31]. Using this precise approach, we investigated the pathogenic properties of human Fus and fly Caz in Drosophila. Individual Fus variants were overexpressed in a tissue-specific fashion using (i) a wing-specific MS1096-Gal4, (ii) a ubiquitous act5C-Gal4 in all fly tissues, (iii) an embryonic nervous system specific Elav-Gal4, and (iv) a larval and adult motor neuron-specific D42-Gal4.

We first found that overexpressing Myc-tagged Fus (Myc-Fus) or FusR521G mutant (Myc-FusR521G) using MS1096-Gal4 interrupted wing growth (Figure 1B-C). The ubiquitous overexpression of Fus severely reduced adult viability at $25^{\circ} \mathrm{C}$ and $19^{\circ} \mathrm{C}$ (Figure $1 \mathrm{G}-\mathrm{H}$ ). Overexpressing Fus in neurons using Elav-Gal4 and D42-Gal4 also significantly reduced adult viability at $25^{\circ} \mathrm{C}$ (Figure $1 \mathrm{G})$, but the adult viability improved at $19^{\circ} \mathrm{C}$ when the Fus overexpression level was lower (Figure $1 \mathrm{H}$ ). The overexpression of Myc-FusR521C produced similar phenotypes to those caused by overexpressing MycFusR521G (data not shown). Surprisingly, we found that expressing Myc-tagged Fus lacking the last 32 amino acids of the NLS (Myc-Fus $\Delta 32$ ) gave rise to a wild-type wing (Figure 1D) and nearly $100 \%$ adult viability (Figure $1 G-H)$. These data give the first indication that the Cterminal NLS is required for Fus toxicity in vivo. 


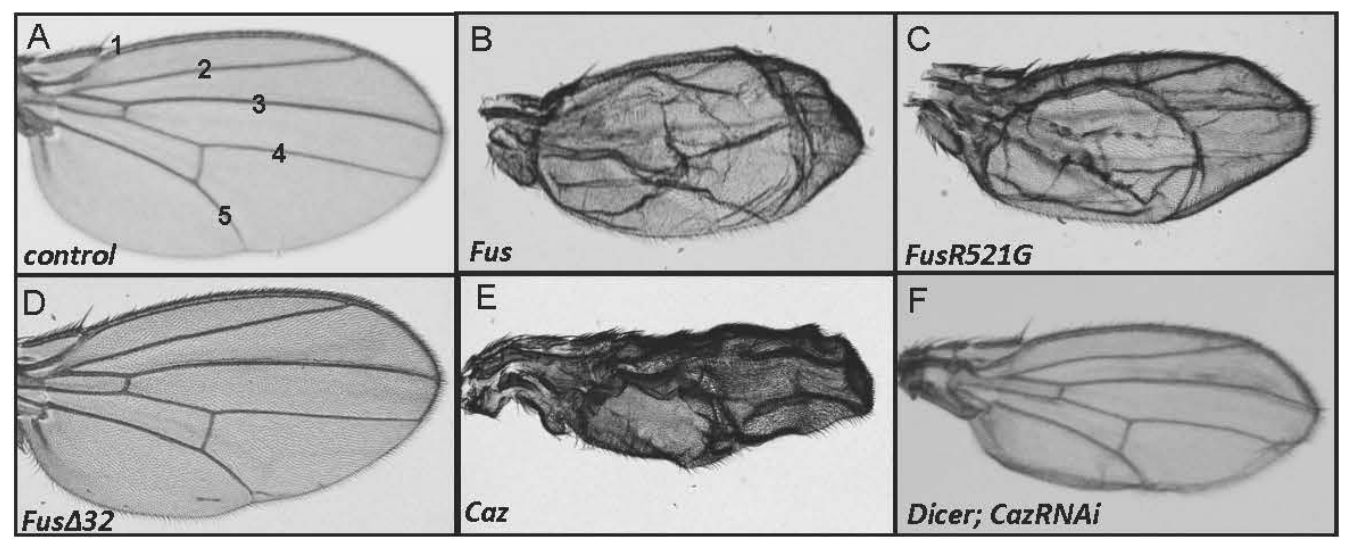

G $\%$ viability at $25^{\circ} \mathrm{C}$
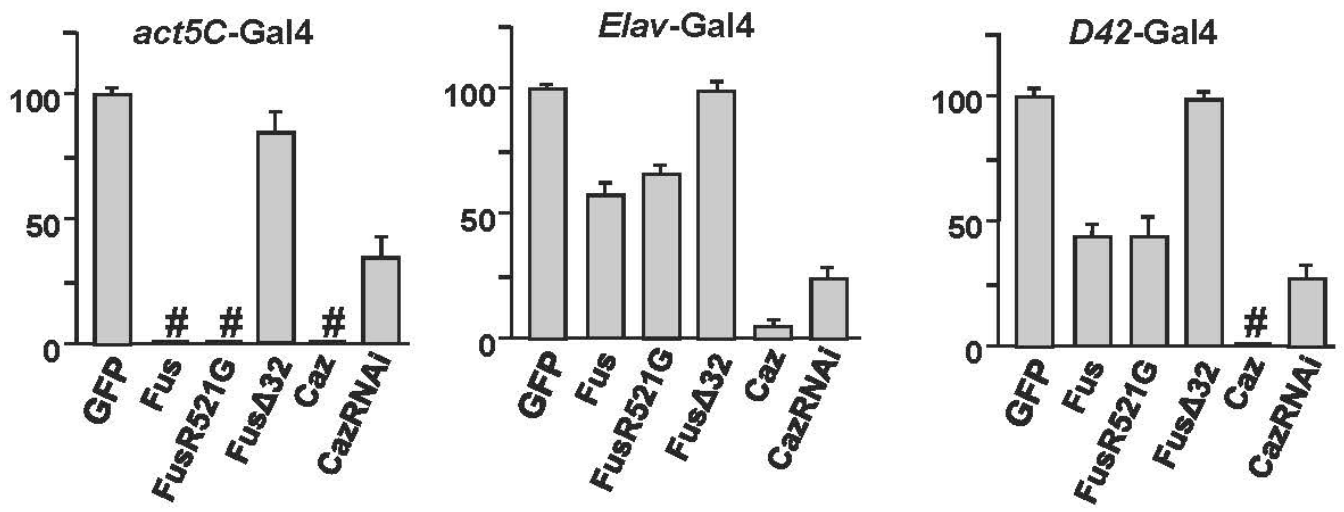

$\mathrm{H}$

$\%$ viability at $19^{\circ} \mathrm{C}$
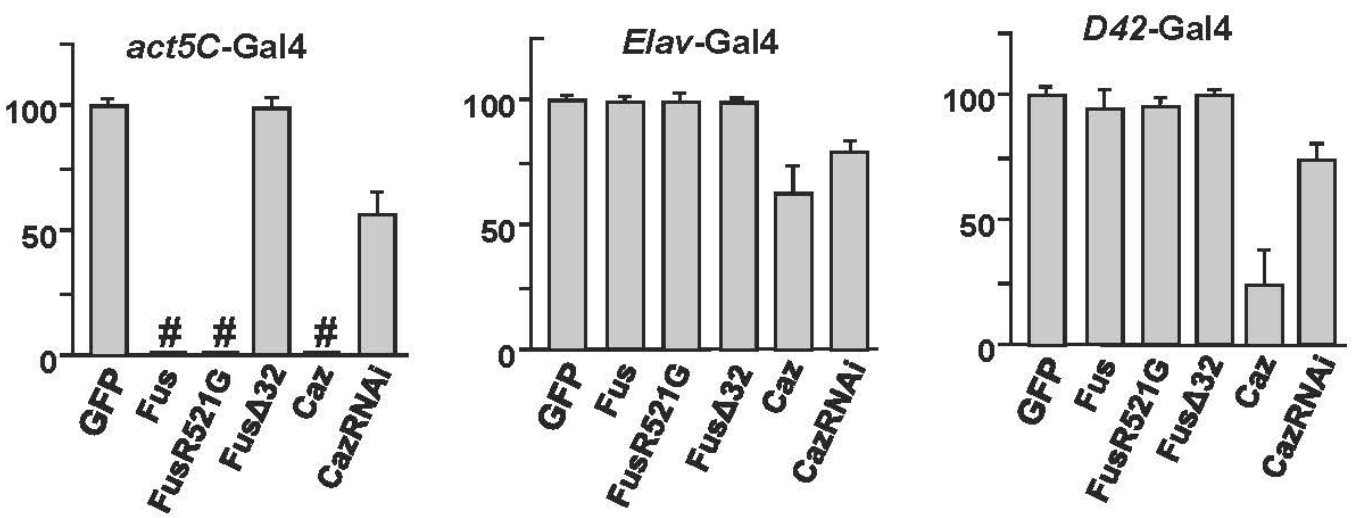

Figure 1 Overexpression of Fus/Caz produces phenotypes and induces growth defects in Drosophila. (A) A wild-type adult wing showing intervein 1-5. (B-F) Adult wings expressing Fus, FusR521G, Fus $\triangle 32$, Caz, or CazRNAi with UAS-Dicer by the wing-specific MS1096-Gal4. (G) Bar graphs showing adult fly viability assayed at $25^{\circ} \mathrm{C}$. (H) Bar graphs showing adult fly viability assayed at $19^{\circ} \mathrm{C}$. Overexpressing the indicated constructs by different Gal4 lines induces growth defects or lethality at larval or pupal stages. The \# indicates lethality at either the larval or pupal stages. 
Caz is the Drosophila homologue of Fus and has been reported to be enriched in the brain and central nervous system $[28,29]$. Caz shares a similar domain structure with Fus (Additional file 1: Figure S1) and exhibits $51 \%$ identity to Fus in the $\mathrm{C}$-terminal region containing the RNA recognition motif and RGG domains. However, its in vivo function and its possible role in neurodegeneration are unclear. We examined the potential role of $\mathrm{Caz}$ in neurodegeneration using overexpression, RNAi knockdown, and knockout approaches in this study. The overexpression of HA-tagged Drosophila Caz (HA-Caz) caused similar phenotypes in the wing (Figure $1 \mathrm{E})$ and even more dramatic decrease in adult viability (Figure $1 \mathrm{G}-\mathrm{H}$ ). The native Caz protein may adopt a more favorable condition in the flies and therefore induce more severe toxicity compared to overexpressed human Fus. These findings suggest that the overexpression of Fus or Caz had similar growth defects in flies. Interestingly, the knockdown of Caz by RNAi affected wing growth (Figure 1F) and reduced adult viability (Figure $1 \mathrm{G}-\mathrm{H}$ ), suggesting that endogenous Caz is involved in Drosophila growth control. This provides initial evidence that Caz deletion can produce toxicity as well, which was thoroughly examined in caz mutant flies later in this study.

\section{Over-expression of Fus/Caz in Drosophila eye induces retinal degeneration}

The Drosophila compound eye, which consists of approximately 750 single eye units called ommatidia, has been used as a model for studying human neurodegenerative disorders [32]. We used a GMR-Gal4 driver to express Myc-Fus, FusR521G, Fus $\Delta 32$, and HA-Caz in Drosophila eyes. We found that the expression of Fus (Figure 2B-C) or Caz (Figure 2H-I) caused severe eye degeneration. The expression of FusR521G induced a phenotype similar to that caused by Fus (Figure 2D-E). We also examined eye structures of the frontal sections using Eosin Phloxine staining. We found that the ommatidia organization was completely lost in fly eyes expressing Fus, FusR521G, or Caz (Figure 2K, L, and $2 \mathrm{~N}$, respectively), compared to eyes from control flies that had an intact structure (Figure 2J). In contrast, flies expressing Fus $\Delta 32$ showed a nearly wild-type eye (Figure 2F-G) and had little, if any, retinal degeneration (Figure 2M)

We noted that expressing two copies of Fus or Caz gave rise to more severe eye phenotypes than expressing one copy (compare Figure 2C and 2I to Figure 2B and $2 \mathrm{H}$, respectively). These observations suggest that the toxicity and phenotypes are clearly dependent on Fus/ Caz expression levels in the Drosophila model system, which supports the hypothesis that the deregulated higher expression of Fus can be toxic. We further found that, in a time course experiment of fly eyes, the overexpression of these proteins produced a progressive eye phenotype, with $\mathrm{Caz}$ being the most toxic protein in eyes (Figure 2O-R) and suggesting that the retinal degeneration was progressive with aging. Western blot analysis was performed and confirmed that the proteins were expressed at comparable levels (Additional file 1: Figure S2). We also found that Myc-FusR521C had a similar effect as Myc-FusR521G did (not shown). These findings suggest that Fus/Caz induces toxicity in a doseand age-dependent manner.

We next systematically characterized the in vivo toxicity of various Fus mutations in different tissues using a variety of Gal4 lines. Table S1 summarizes the results of wing, eye, and adult viability results from the above Fus/ Caz constructs and two additional mutations. Sequence analysis revealed a highly conserved tyrosine kinase phosphorylation motif (RXXPY) in the C-terminus of Fus/Caz, and mutation of the tyrosine residue in this motif (FusY526F) significantly reduced the in vivo toxicity of Fus (Table S1). A consistent but striking finding in these experiments is that the truncation mutation Fus $\Delta 32$ lacking the C-terminal NLS was largely nontoxic in all lines we tested. We generated a shorter truncation mutation of Fus lacking the RGG-rich domain in the C-terminus (Fus1-453) and found that it was also non-toxic and thus allowed full adult viability (Table S1, data not shown).

\section{The expression of Fus/Caz in motor neurons causes locomotive impairment}

We next characterized the locomotive function of the flies overexpressing Fus, Fus mutants, or Caz. We used another motor neuron-specific OK371-Gal4, which is expressed earlier than D42-Gal4 in the larval stage. When Fus, FusR521G, or Caz was overexpressed through the OK371-Gal4 line, the locomotive ability of third instar larvae was markedly impaired (Figure 3A). The average moving distances also correlated with the protein toxicity presented in Table S1. The Caz larvae moved the shortest distance and the Fus $\Delta 32$ larvae moved the longest distance in a specific period of time among the flies expressing different forms of Fus/Caz (Figure 3A). A comparable level of protein expression in larval brain extracts was confirmed by Western blot (Figure 3B).

We further examined the locomotion of adult flies expressing Fus, Fus mutants, and Caz. We used the other motor neuron-specific Gal4 line, D42-Gal4, in the adult movement assays because Fus, FusR521G, and Caz expression by OK371-Gal4 caused pupal lethality. We found that the locomotive ability of two-day-old flies was severely impaired by the expression of Fus or FusR521G but not by the expression of Fus $\Delta 32$ (Figure 

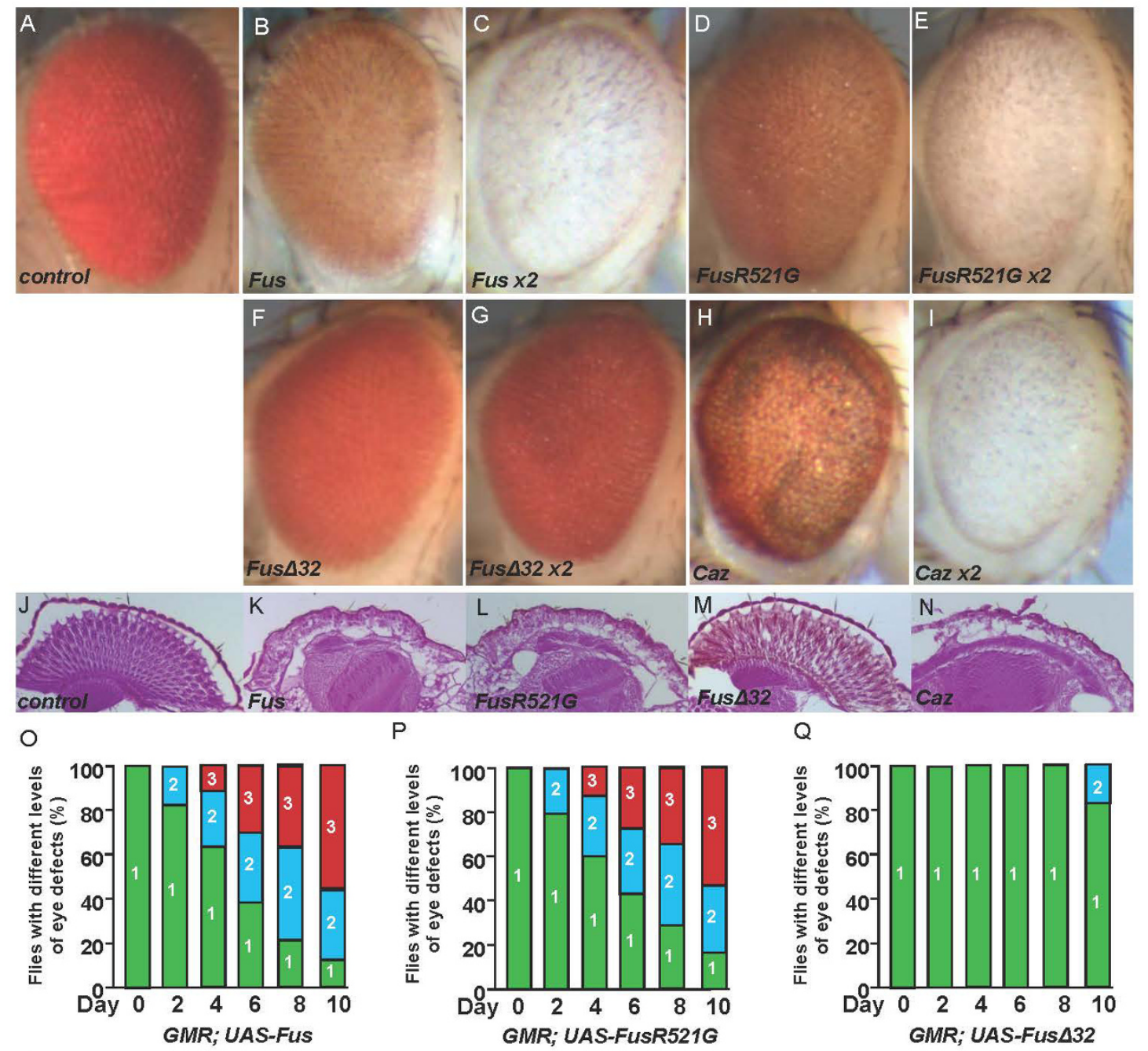

$\mathrm{R}$
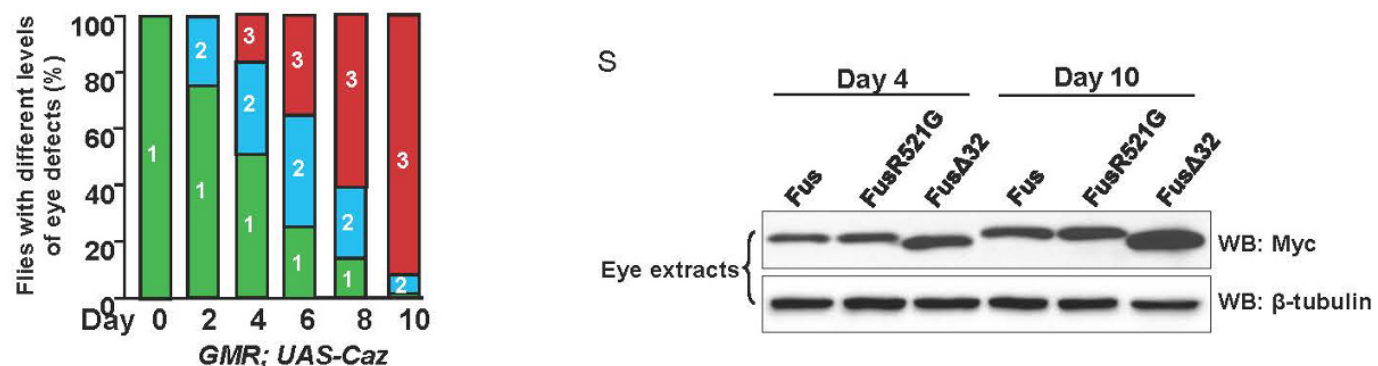

Figure 2 Expression of Fus/Caz in fly eyes leads to progressive eye defects in a dose- and age-dependent manner. (A) A wild-type eye showing the typical ommatidia structure. (B-I) Morphology of eyes expressing one or two copies of Fus, FusR521G, Fus $\triangle 32$, or Caz by GMR-Gal4 at day 4 after adult hatching. Fus, FusR521G, and Caz expressing eyes exhibit ommatidia loss and necrotic lesions. (J) Retinal section of a wildtype fly eye stained with Eosin shows intact ommatidial and rhabdomere structure. (K-N) Retinal sections of eyes expressing two copies of Fus, FusR521G, Fus $\triangle 32$, or Caz by GMR-Gal4 at 4 DAE were stained with Eosin. Rhabdomere structures were disintegrated and ommatidia were disorganized in eyes expressing Fus, FusR521G, or Caz, but not in eyes expressing Fus $\triangle 32$. (O-R) Quantification of the eye defects when expressing the indicated constructs during aging. Eye defects were classified in three levels: 1, <30\% ommatidia loss; 2, 30-70\% ommatidia loss, and 3, > 70\% ommatidia loss. The percentages of flies with each level of eye defects are shown in different colors (1, green; 2, blue; 3, red). (S) Western blots were performed with eye extracts from flies in Figure 2O-R at the indicated time points to monitor the levels of protein expression. Each individual protein was expressed at the attP site of the 75B1 locus by GMR-Gal4 to ensure that the proteins were expressed at a comparable level. 
A

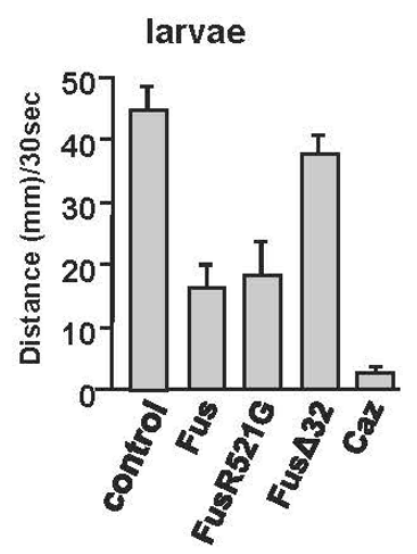

C

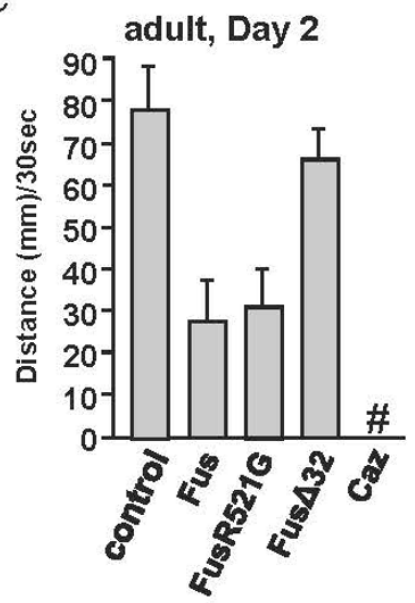

B

\section{Larval brain extracts}
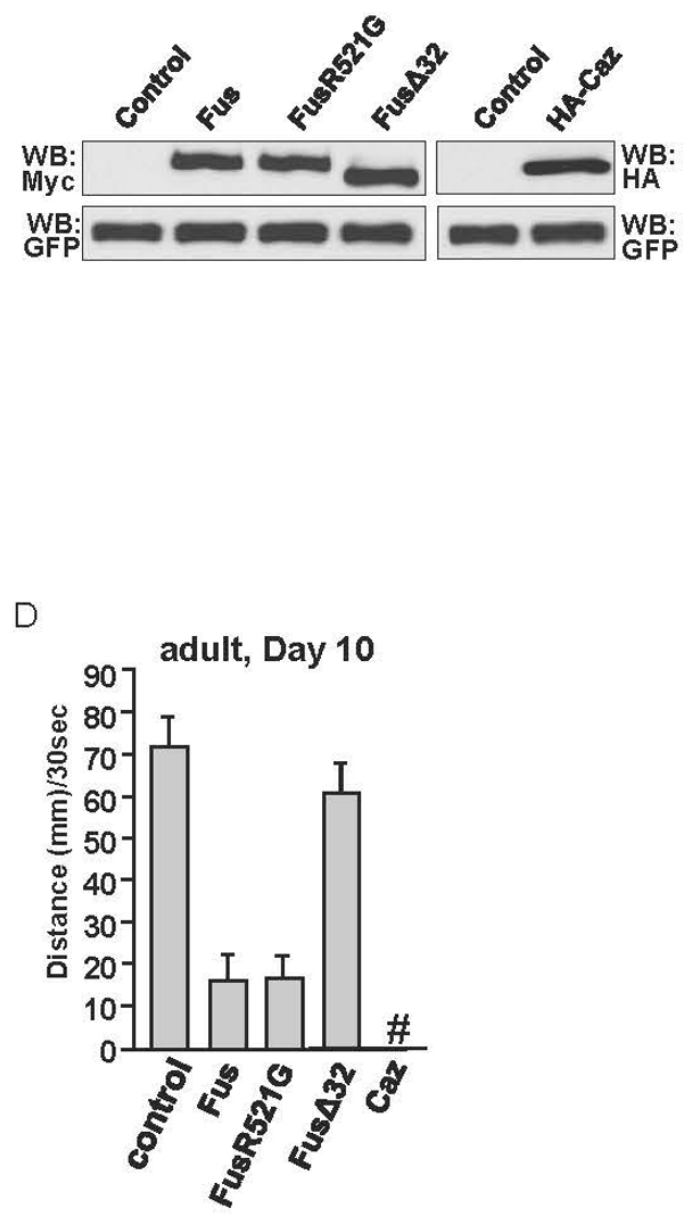

Figure 3 The locomotive ability affected by the expression of different forms of Fus or Caz. (A) Expression of Fus/Caz in fly motor neurons impairs the locomotive ability in larvae. A graph of the average moving distance of the third instar larvae traveling over 30 sec is shown here ( $n=15$ male larvae scored for each group). Each larva was tested 3 times. The OK371-Gal4 was used for these experiments. (B) Larvae brains with VNCs from experiments in A were subjected to Western blots with antibodies against the epitope tags to monitor the comparable levels of protein expression. GFP serves as an internal protein expression and loading control. (C-D) Graphs of average climbing distance of the adult flies at Day 2 or Day 10 after emergence. Each adult fly was placed at the bottom of a $2 \mathrm{ml}$ pipette and allowed to climb vertically for $30 \mathrm{~s}$ ( $n=20$ male flies for each group). Each fly was counted 3 times. The height that the flies climbed on the inside wall of the pipette is an indication of their locomotion ability. The D42-Gal4 was used for these experiments. The "\#" symbol indicates pupal lethality. The larval locomotion was significantly impaired by Fus, FusR521G, and Caz expression, but not by Fus $\triangle 32$ expression.

3C). Similar results were achieved by analyzing the tenday-old flies (Figure 3D). In this experiment, the expression of Caz by the D42-Gal4 caused pupal lethality, indicating that Caz induced severe toxicity (Figure 3C-D). We also found that the expression of FusR521C produced a similar phenotype as that of FusR521G (data not shown). Taken together, the locomotive defects caused by Fus or Caz suggest that Fus/Caz is involved in neurodegeneration after larval stage.
Overexpression of Fus/Caz disrupts presynaptic terminals at the neuromuscular junction (NMJ) and causes disorganization of motor neurons in larval ventral nerve cord (VNC)

To gain more insights into the disease etiology, we analyzed the morphology of motor neuronal presynaptic terminals at the NMJ in flies. Third instar larval muscle fillets were dissected and double-labeled with an antiHRP antibody to visualize the neuronal membrane and 
an anti-Dlg antibody to mark the subsynaptic reticulum surrounding each bouton. The expression of a membrane GFP (mGFP) by OK371-Gal4 was used to mark the motor neurons and did not have any effect on bouton formation at NMJ (Figure 4A, F and 4I).
Morphological abnormalities were found in larvae coexpressing mGFP with either Fus or Caz by OK371Gal4. As shown in Figure 4, the overexpression of Fus substantially reduced the area (Figure 4B) and the number of both large and small boutons (Figure 4F and 4I).
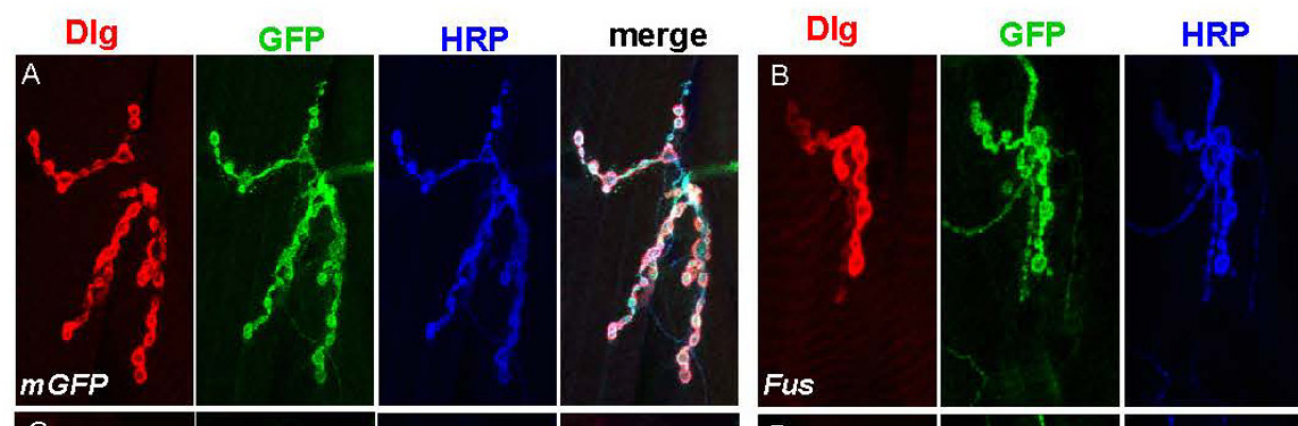

\section{merge}
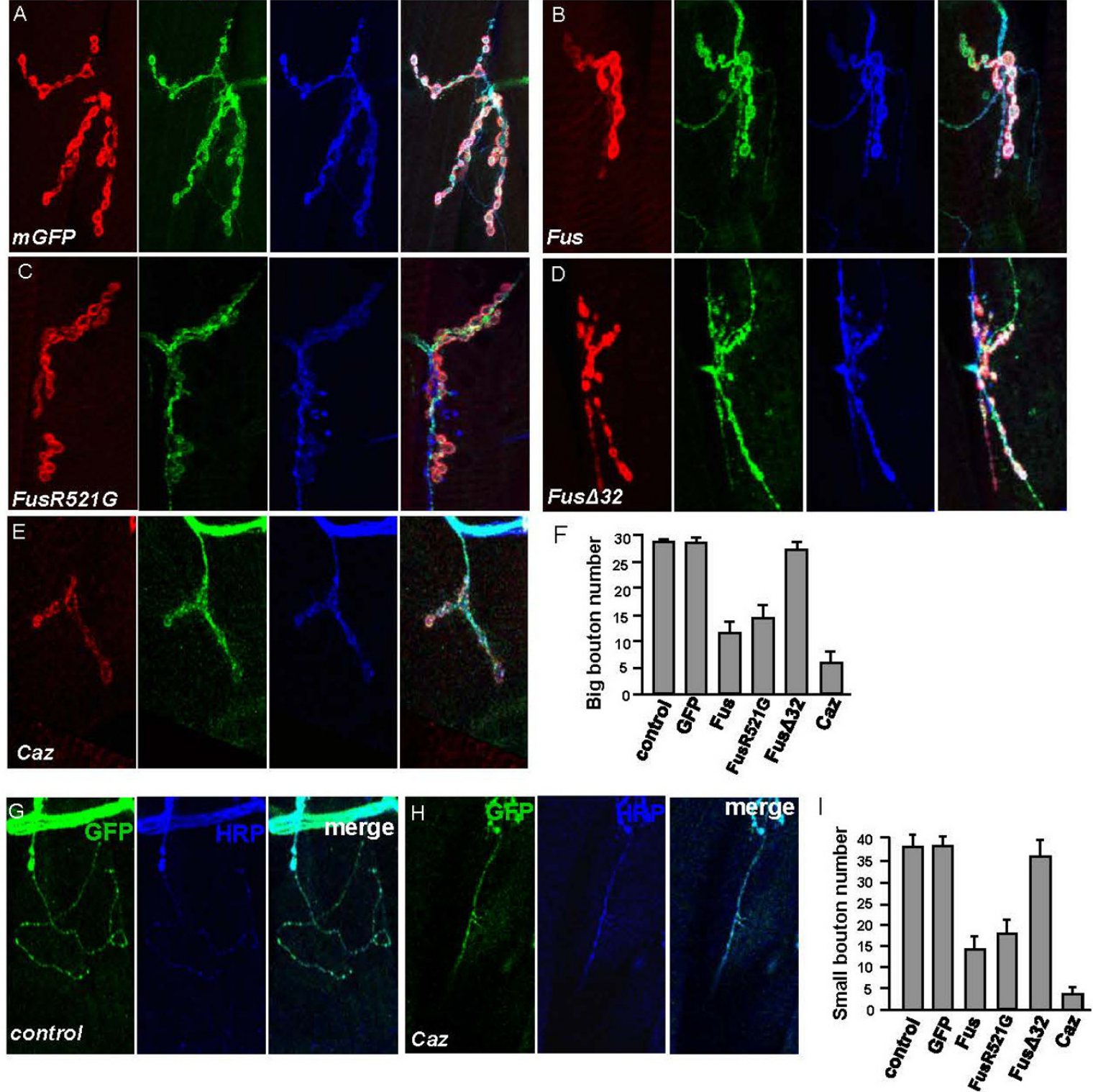

Figure 4 The overexpression of Fus/Caz causes morphological and functional defects in NMJs. (A) A representative confocal image (projection over the $Z$ axis) of muscle 6/7 NMJs in the abdominal segment A2 of a third instar larva expressing mGFP by OK371-Gal4. The antiHRP antibody staining labels the neuronal membrane and the anti-Dlg antibody staining marks the subsynaptic reticulum surrounding each bouton. (B-E) NMJs in the abdominal segment A2 from larvae coexpressing mGFP with Fus, FusR521G, Fus $\triangle 32$, or Caz by OK371-Gal4 were stained for Dlg, HRP, and Myc or HA with the appropriate antibodies. Branches and the number of large boutons revealed by Dlg staining were dramatically reduced by Fus, FusR521G, and Caz, but not by Fus $\triangle 32$ expression. (F) Quantification analysis of the large boutons in the NMJs from ventral longitudinal muscles 6 and 7 of segment A2 (mean \pm s.d.; $n \geq 20$ ). OK371-Gal4 alone served as control. (G-H) Representative images of axon branches and small boutons, as visualized by both mGFP and HRP staining, at muscle 6/7 NMJs in the abdominal segment A2. (I) Quantification of the small boutons in the NMJs from ventral segment A2 (mean \pm s.d.; $\mathrm{n} \geq 20$ ). OK371-Gal4 alone served as control. 
Compared to Fus, expression of Caz had more severe NMJ defects (Figure 4E, F, H, and 4I). Interestingly, overexpression of Fus $\Delta 32$ induced little, if any, disruption in the presynaptic terminals (Figure 4D, F, and 4I). These findings are consistent with the locomotive deficiency observed in the animals (Figure 3C-D), suggesting that the abnormal NMJs in flies expressing Fus are responsible for the impaired motor function. The results are also consistent with the toxicity of Fus/Caz observed in other tissues (Additional file 1: Table S1).

To further elucidate whether Fus overexpression caused any defects in motor neuron cell bodies, we next examined the motor neurons in larval ventral nerve cord (VNC) from late third instar larvae expressing different Fus/Caz proteins by the OK371-Gal4. Motor neurons in the ventral clusters are well-organized in the larval VNC in wild-type flies (Figure 5A). In addition, longitudinal motor neurons, which ensheath the cortex of the VNC, are logitudinally connected, and the segmental nerve of the abdominal neuromeres A1-A5 are well-organized in wild-type flies (Figure 5B). As expected, we found that the OK371-Gal4-mediated expression of Fus, FusR521G, or Caz induced disorganized motor neurons in A1-A5 of the larval VNC (Figure $5 \mathrm{C}, \mathrm{D}$, and $\mathrm{F}$ ). In contrast, the overexpression of Fus $\Delta 32$ again had no effect on the organization of neuromeres A1-A5 (Figure 5E), which was consistent with the finding that this truncation mutant had no toxicity in motor neurons (Figures 3 and 4).

To solidify the relationship between nuclear localization and toxicity, we constructed Myc-tagged FusNES with a typical nuclear export signal (NES) fused in frame to the C-terminus of FUS (Myc-FusNES) and Myc-tagged chimeric Fus $\triangle 32$ NLS with the NLS of another RNA binding protein hnRNP D fused to the Cterminus of Fus $\Delta 32$ (Myc-Fus $\Delta 32 \mathrm{NLS}$ ). We then generated transgenic lines at the 75B1 - VK5 attP locus. Expression of FusNES by the OK371-Gal4 had markedly less effect in larva VNC (Figure 5G) and did not induce locomotive defects in the third instar larvae (Figure 5I). Thus, both Fus $\Delta 32$ and FusNES were largely non-toxic in a similar fashion. In contrast, expression of Fus $\triangle 32 N L S$ induced disorganization of motor neurons in VNC (Figure $5 \mathrm{H}$ ) and caused locomotive defects at a comparable level as in flies expressing Fus (Figure 5I). We also found that FusNES did not cause any phenotype in the eyes or NMJs when driven by GMR-Gal4 or OK371-Gal4, whereas Fus $\Delta 32$ NLS did (Table S1).

We examined the subcellular localization of Fus/Caz proteins using confocal microscopy. Consistent with our previous findings in mammalian cells [19], we found that Fus, Caz, and Fus $\Delta 32$ NLS were largely localized in the nuclei of motor neurons in the VNC (Additional file 1: Figures S2A and S2D). There was a small increase in cytoplasmic staining of FusR521G (Additional file 1: Figure S2B), whereas Fus $\Delta 32$ and FusNES were largely cytosolic (Additional file 1: Figure S2C and S2E). Taken together, the data from all tissues examined (Figures 1, $2,3,4)$ suggest that the toxicity of Fus/Caz in flies is likely due to, at least in part, the abnormal function caused by the nuclear fraction of Fus/Caz protein.

\section{The expression of Fus/Caz induces apoptosis in motor neurons}

In the above experiment, we observed an abnormal nuclear structure in motor neurons overexpressing Fus/ Caz when the cells were stained with Hoechst 33342 nuclear dye. Motor neuron cells expressing the mGFP alone showed a normal even nuclear staining (arrow in Figure 6A), which was almost identical to the structure of the adjacent non-motor neuronal cells (arrowhead in Figure 6A). Surprisingly, the overexpression of Fus or Caz with mGFP resulted in decreased nuclear staining reminiscent of apoptotic bodies in the GFP-positive motor neurons (arrows in Figure $6 \mathrm{~B}$ and $6 \mathrm{E}$ ). In contrast, the nuclear staining was largely uniform in the adjacent non-Fus/Caz-expressing cells (arrowheads in Figure $6 \mathrm{~B}$ and $6 \mathrm{E}$ ). Consistent with the earlier data, the expression of FusR521G gave rise to a similar effect to that of Fus (arrow in Figure 6C). The expression of Fus $\triangle 32$ and FusNES gave rise to normal nuclear staining in motor neurons (arrows in Figures $6 \mathrm{D}$ and $6 \mathrm{E}$ ), whereas Fus $\Delta 32$ NLS induced abnormal nuclear staining (arrow in Figure 6G). These data again suggest that the toxicity of Fus/Caz is caused, at least in part, by the protein localized in the nucleus.

We next performed the terminal deoxynucleotodyl transferase dUTP nick end labeling (TUNEL) assay to examine whether the expression of Fus/Caz could induce apoptosis in the VNC. We did not detect any TUNELpositive cells in control third instar larval VNC (Figure $6 \mathrm{H})$. However, Fus expression in VNCs led to approximately 20-30 TUNEL-positive cells in the same larval stage (Figure 6I), suggesting that overexpression of Fus induced neuronal apoptosis in the VNC. In contrast, the overexpression of Fus $\Delta 32$ or FusNES did not give rise to TUNEL-positive cells (Figure 6J, not shown), which was consistent with the finding that Fus $\triangle 32$ and FusNES induced little disorganization of motor neurons in the VNC. In addition, we found that the overexpression of Caz induced approximately 40-50 TUNEL-positive cells in the VNC (Figure 6K), which could explain why the overexpression of Caz at an equivalent level as Fus led to a more severe toxicity. Our results suggest that the neurodegeneration induced by Fus/Caz overexpression occurs due to apoptosis in the Drosophila VNC. 


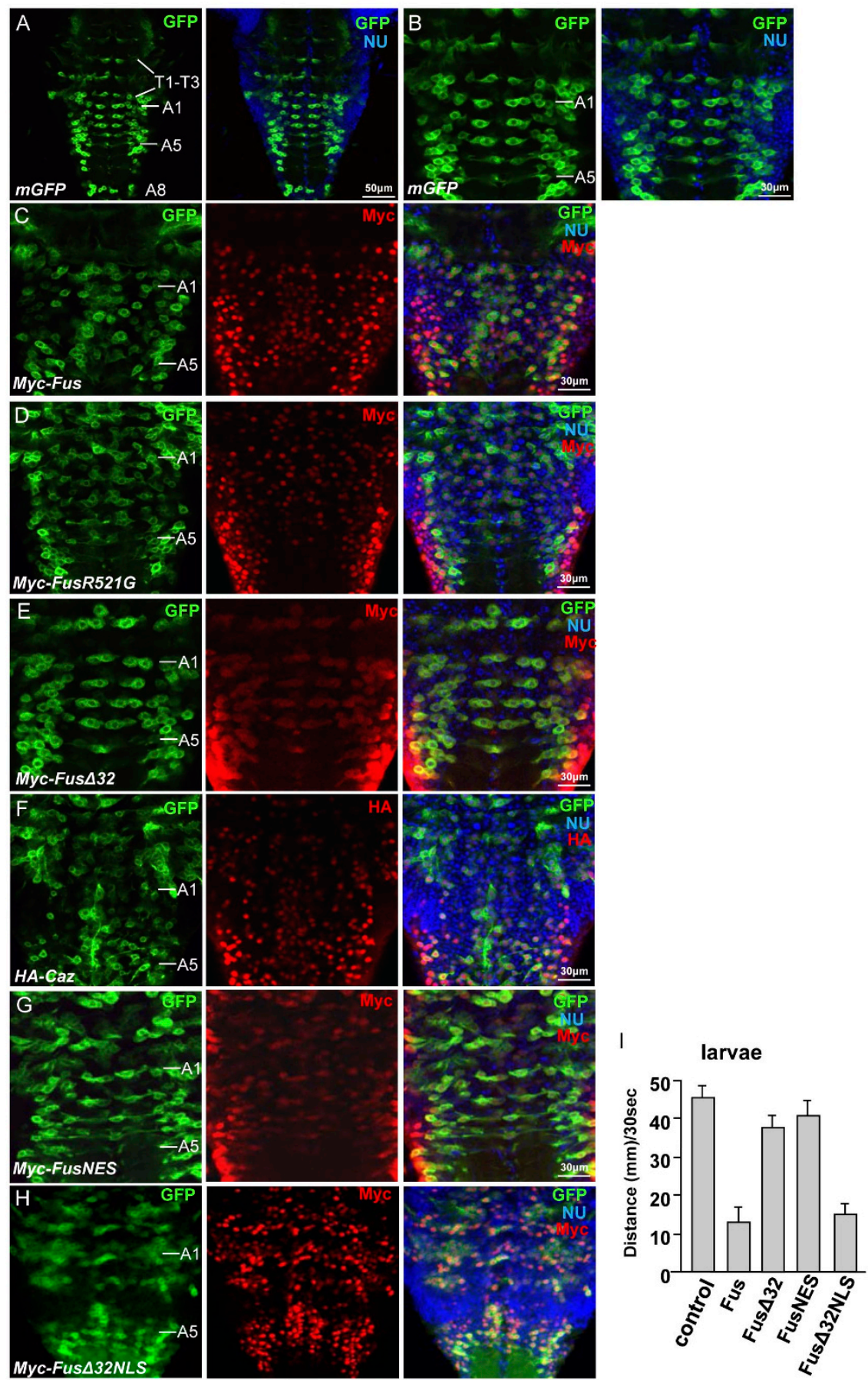

Figure 5 The expression of Fus/Caz disrupts the arrangement of motor neurons in the VNC. (A) A VNC from the third instar larva expressing mGFP by OK371-Gal4 was stained with nuclear dye Hoechst 33342 (blue NU signal). The GFP signal indicates intact motor neurons. T1-T3 indicates the three thoracic neuromeres and A1-A8 indicates the eight abdominal neuromeres. (B) Shown here are the well-organized segmental motor neurons of the abdominal neuromeres A1-A5. The organization of motor neurons is used as a model to examine the neuronal toxicity of Fus/Caz in this study. (C-E) VNCs from third instar larvae co-expressing mGFP with Myc-tagged Fus, FusR521G, or Fus $\triangle 32$ by OK371Gal4 were stained with an anti-Myc antibody and Hoechst. Fus and FusR521G disrupted the organization of motor neurons in A1-A5 neuromeres, but Fus $\triangle 32$ did not. (F) A VNC from larva coexpressing mGFP with HA-Caz was stained with an anti-HA antibody and Hoechst 33342. The expression of Caz severely disrupted the arrangement of motor neurons in A1-A5 neuromeres. (G-H) VNCs from larvae coexpressing mGFP with Myc-FusNES or Myc-Fus $\triangle 32 N L S$ were stained with the anti-Myc antibody and Hoechst 33342. (I) A graph of the average moving distance of the third instar larvae expressing the indicated proteins and traveling over $30 \mathrm{sec}(\mathrm{n}=15$ male larvae scored for each group). Each larva was tested 3 times. The OK371-Gal4 was used as the driver. 

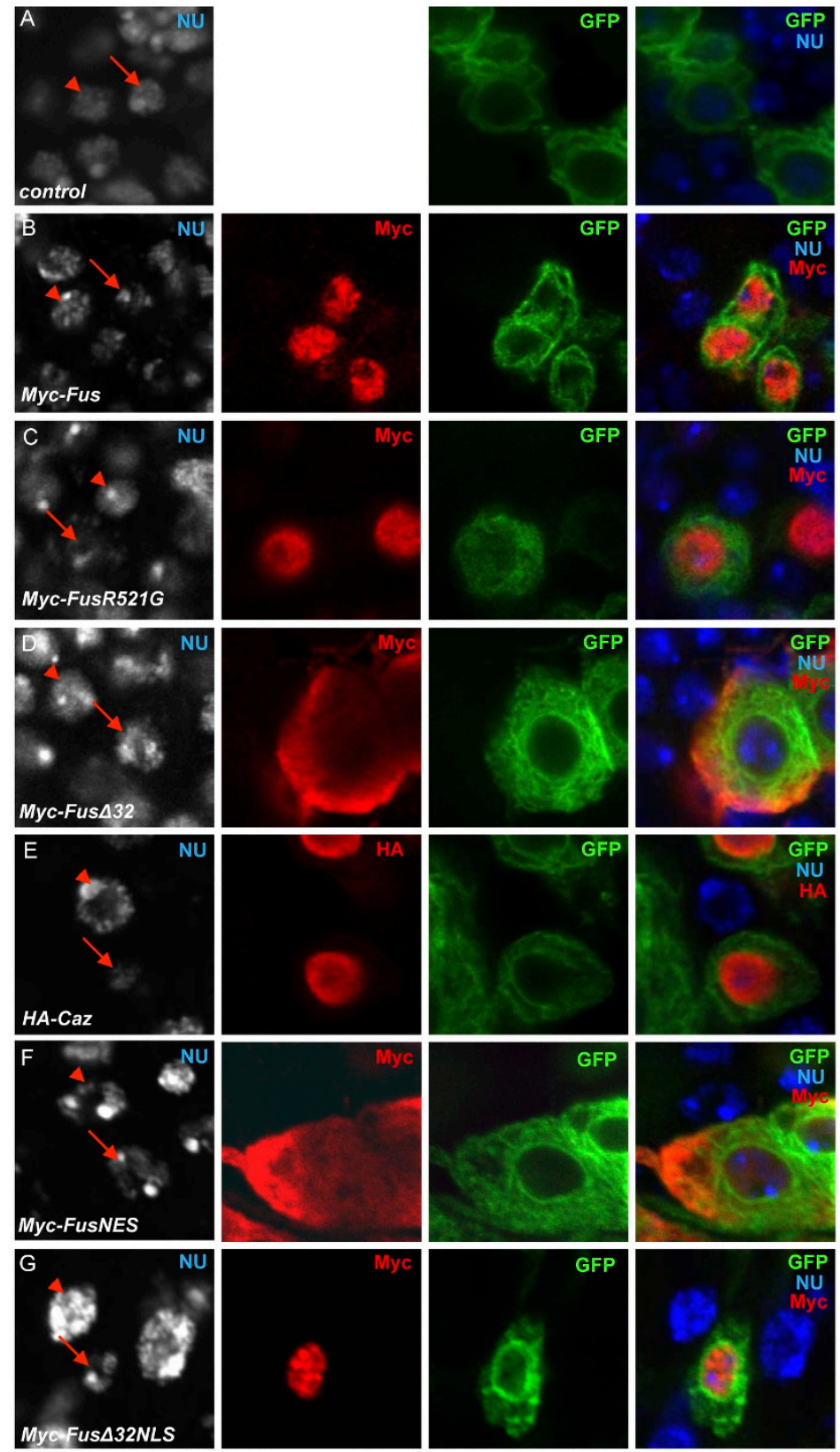

Hy
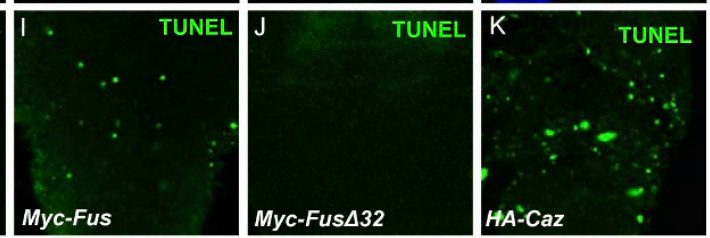

Figure 6 Neurodegeneration induced by Fus/Caz overexpression occurs due to apoptosis. (A) A large magnification of the VNC from a third instar larva expressing mGFP by OK371-Gal4 shows GFP-labeled motor neurons and adjacent non-motor neuronal cells. Arrows in A indicate the nuclear structure of motor neuronal cells and arrowheads indicate the nuclear structure of adjacent cells. (B-G) VNCs from larvae coexpressing mGFP with Myc-Fus, Myc-FusR521G, HA-Caz, Myc-FusNES, or Myc-Fus 32 NLS were stained with the indicated antibodies and the Hoechst 33342 dye to label the nuclear structures. Arrows in B, C, E, and G indicate the decreased nuclear staining and disrupted nuclear structure by the expression of Fus, FusR521G, Caz, or Fus $\triangle 32$ NLS in the nuclei. Arrows in D and F indicate the unaffected nuclear structures. The arrowheads indicate the nuclear staining of normal adjacent cells, which serves as a control. (H-K) TUNEL assays were performed on the VNC from the larvae of OK371-Gal4 (control) or the larvae expressing Fus, Fus $\triangle 32$, or Caz by OK371-Gal4. Apoptotic cell death is measured by the TUNEL signal (green) 
The phenotypes of caz deletion mutant Drosophila

Our initial study of caz inactivation in flies using the RNAi lines from the Vienna Drosophila RNAi Center (VDRC) found that CazRNAi caused a defective morphology in the wing (Figure 1F) and reduced the viability of adult flies (Figure 1G-H). Next, we explored the neuronal function of $\mathrm{Caz}$ by analyzing caz mutant phenotypes. A deficiency line $D F(1) B S C 759$, which harbors a deletion in $c a z$ and other genes upstream of $c a z$, was first used. $D F(1) B S C 759$ heterozygous females exhibited wild-type eyes with the balancer marker (Figure 7B), but $D F(1) B S C 759$ homozygous females were lethal at the pupa stage and the adult escapers exhibited loss of ommatidia in the eyes (Figure $7 \mathrm{C}$ and Additional file 1: Figure S3C). DF(1)BSC759 males also showed a similar eye phenotype (Figure 7E and Additional file 1: Figure S3E). These data suggest that the lack of Caz disrupts the formation of Drosophila ommatidia. Another deficiency line $D F(1) E D 7355$, which retains the wild-type $c a z$ gene but lacks a fragment upstream of $c a z$, was able to suppress the eye phenotypes in the complementary test. The results suggest that the phenotype in $D F(1)$ BSC759 was caused by the loss of caz but not other genes. We also examined the other caz mutant allele caz1 [27] and found that caz1 mutant had similar phenotypes as $D F(1) B S C 759$.

We next examined how caz deletion caused the degenerative phenotypes in eyes as seen in the caz homozygous flies (Figure 7C', compared to Figures 7A' and $\left.7 \mathrm{~B}^{\prime}\right)$. An anti-HRP antibody was used to detect the photoreceptor neurons and an anti-Elav antibody to detect the pan neurons in third instar larvae. The Elav signal was evenly distributed in the well-organized ommatidia units of eye discs in the wild-type (Figure 7F-F') and $c a z$ heterozygous female (Figure 7G-G') flies. In contrast, the eye discs from caz/caz homozygous female (Figure 7H-H') and caz/y male (Figure 7I-I') flies showed a substantially decreased Elav staining with abnormal puncta in disorganized ommatidia. In addition, Caz knockdown by RNAi also disrupted ommatidia formation (Figure 7Q). These data suggest that Caz is required for maintaining normal function of the neurons in the eye.

We next assessed the function of Caz in Drosophila motor neurons. Loss of $c a z$ in motor neurons significantly impaired the locomotive ability of adult flies of both males and females (Figure 7J) and shortened the lifespan of adult flies (data not shown). Male caz mutant larvae showed severe reduction in locomotive activity (Figure $7 \mathrm{~K}$ ). Examination of the NMJs in the male mutant larvae revealed a severe decrease in numbers for both big and small boutons in the NMJs (Figure 7L-M). The eye phenotype was fully rescued by expressing wild-type Fus or Caz (data not shown), which is consistent with the findings in fly locomotive function rescue from a previous study [27]. These results suggest that $\mathrm{Caz}$ is required for the function of photoreceptor neurons as well as motor neurons.

We further assessed the organization of the motor neurons in VNC in caz mutant flies as we did in the Fus/Caz overexpressing flies. We found that caz mutant male flies exhibited disorganized motor neurons in neuromeres A1-A5 of the VNC (Figure 7N), a phenotype similar to that caused by the overexpression of Fus/Caz (Figure 5C and 5F). However, unlike the Fus/Caz overexpression flies, loss of $c a z$ did not cause any decrease in nuclear staining by Hoechst 33342 (arrow in Figure 7O). Mutating caz in the motor neurons also did not produce any TUNEL-positive cells in the VNC (Figure 7P). These results suggest that the loss of caz did not promote cell death of motor neurons although it disrupted NMJs and impaired locomotive function in the caz mutant flies. Consistent with these results, Caz RNAi also interrupted the arrangement of motor neurons in VNCs (Figure 7R), but did not cause any decrease in nuclear staining (Additional file 1: Figure S7) or any TUNEL-positive cells (not shown). Collectively, these data suggest that the mechanism underlying the phenotypes in the caz mutant line is different from that in the Fus/Caz overexpression flies.

\section{Discussion}

The vast majority of the molecules critical to neuronal function are conserved between Drosophila and more complex vertebrate systems, thus Drosophila is a powerful model to study neurodegenerative diseases including ALS. In this study, we showed that the overexpression of human Fus as well as the Drosophila homolog Caz caused neuronal toxicity in vivo in all Gal4 lines that we tested, including ubiquitous expression (act5C-Gal4), wings (MS1096-Gal4), eyes (GMR-Gal4), neurons (ElavGal4) and motor neurons (D42- and OK371-Gal4). Since Fus/Caz protein was expressed at late larval or pupal stages and the degenerative phenotypes were progressive with aging, the Fus/Caz overexpressing flies are a reasonably good model to study the relevance of Fus in motor neuron degeneration in ALS. Furthermore, we provide evidence that both loss of Caz and overexpression of Fus/Caz can induce phenotypic defects although the underlying cellular mechanisms are likely to be different in these two models.

\section{Nuclear toxicity of Fus/Caz in the absence of cytoplasmic inclusions}

It has been recently shown that the overexpression of Fus in flies leads to neuronal toxicity [25-27]. However, it is unknown whether overexpression of Drosophila Caz can cause toxicity. In this study, overexpression of 

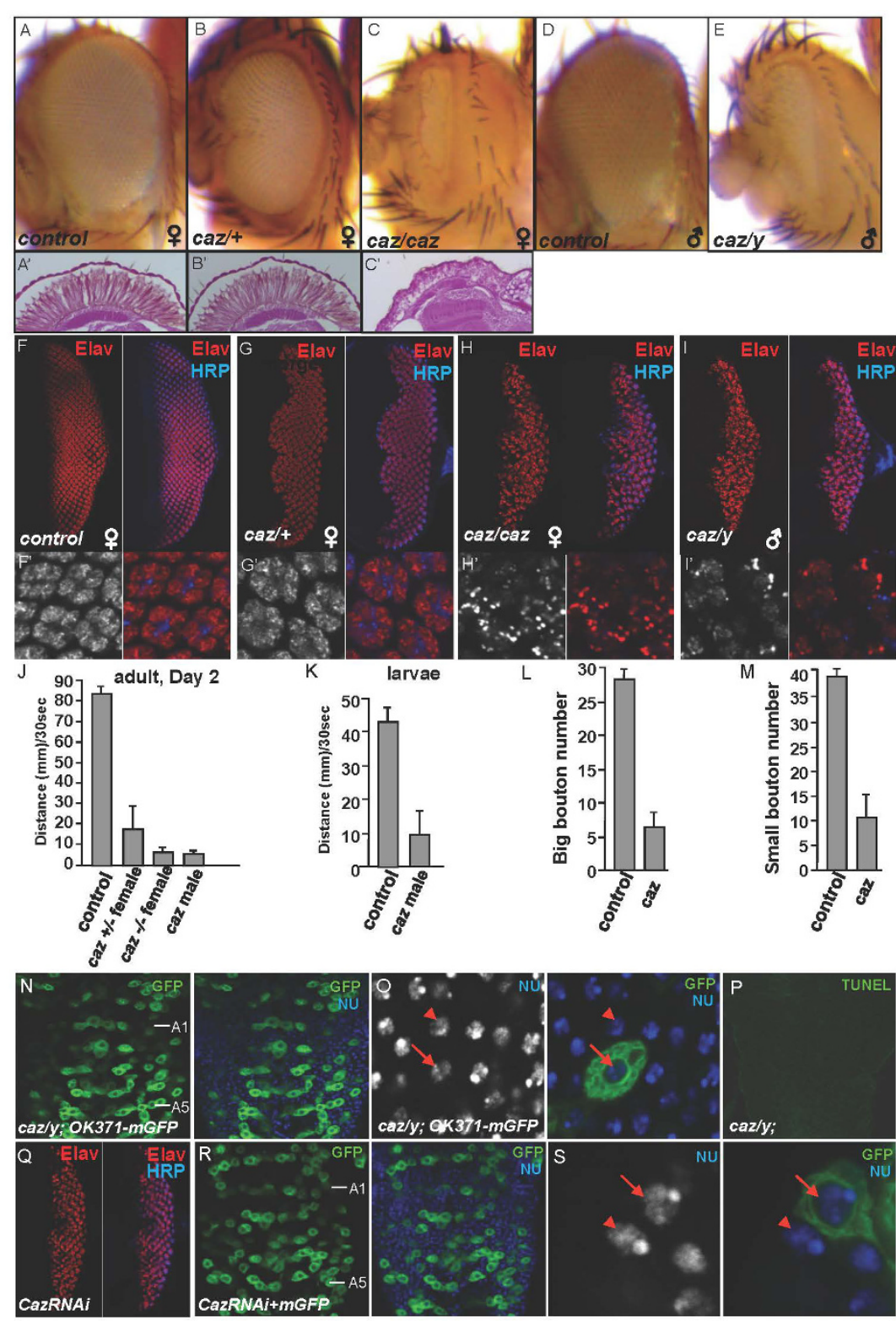

Figure 7 The loss of $c a z$ leads to neuron functional defects in Drosophila. (A-A') An eye from yw female flies showing wild-type ommatidial and rhabdomere structure. (B-B') An eye from caz heterozygous female flies shows the balancer marker and intact ommatidial and rhabdomere structure. (C-C') An eye from caz homozygous female fly shows the loss of ommatidia and the disrupted rhabdomere structure. (D) An eye from yw male fly showing normal ommatidial. (E) An eye from caz mutant male showing the loss of ommatidia. (F-F') A wild-type eye imaginal disc from the third instar larvae was stained for Elav and HRP to show the well-developed and well-organized structure of the ommatidia units. (G-H') Eye discs from either caz heterozygous or homozygous female third instar larvae were immunostained for Elav and HRP. (III') An eye disc from caz male larva was stained for Elav and HRP. (J) A graph of the average climbing distance of caz mutant adult flies at Day 2 after emergence. The same method was used as in Figure 4. $\mathrm{N}=20$ flies for each genotypic group. (K) A graph of the average moving distance of the third instar larvae of caz males traveling over $30 \mathrm{~s}$ ( $\mathrm{n}=15$ male larvae scored for each group). Each larva was tested 3 times. (L-M) Quantification analysis of the large and small boutons in the NMJs from ventral longitudinal muscles 6 and 7 of segment A2 in caz mutant male larvae (mean \pm s.d.; $\mathrm{n} \geq$ 20). (N) A VNC from a caz mutant male fly expressing mGFP by OK371-Gal4 shows the disrupted motor neuron organization in A1-A5 neuromeres. $(\mathrm{O})$ A large magnification image from $\mathrm{N}$. The arrow indicates the motor neuron nuclear staining, which is similar to the nuclear staining of an adjacent cell (arrowhead). (P) TUNEL assay was performed on VNC from the larvae of a caz/+ female, caz/caz female, and caz male. A representative TUNEL image from the VNC of a caz male is shown here. None of the caz mutant larvae showed TUNEL-positive cells. (Q) An eye imaginal disc expressing Dicer and CazRNAi by GMR-Gal4 was stained for Elav and HRP. (R) A VNC from a third instar larva coexpressing mGFP with Dicer and CazRNAi shows the disorganization of motor neurons in A1-A5 neuromeres. (S) A large magnification image from R. The arrow indicates the motor neuron nuclear staining, which is similar to the nuclear staining of an adjacent cell (arrowhead). 
fly Caz caused similar or even more severe toxicity in all experiments compared to overexpression of human Fus (Figures 1, 2, 3, 4). This is reminiscent of the studies of another ALS-implicated RNA binding protein TDP-43 that the overexpression of TDP-43 was shown to be toxic in yeast [33], Drosophila [23,24,34,35], C. elegans [36], and mouse $[37,38]$. It is noted that protein inclusions of wild-type Fus have also been reported in human ALS patients $[7,8]$. These findings underscore the importance of understanding the underlying molecular mechanisms of Fus in ALS.

Our group and others have shown that the last 32 amino acids of the protein contain an NLS that is both essential and sufficient for nuclear targeting. It has also been shown that the ALS-related mutations in Fus increased the cytoplasmic localization of the protein and promoted the co-localization of Fus with stress granules in the cytosol [19-21]. However, the cytoplasmic accumulation may not be essential or sufficient for the toxicity observed for the protein $[39,40]$. Thus, the link between toxicity and subcellular localization of Fus needs to be characterized in vivo.

A novel finding of this study is that Fus nuclear localization is required for its toxicity in Drosophila. The overexpression of Fus $\Delta 32$ at comparable levels consistently showed little or no toxicity in all tissues. The in vivo subcellular localization of wild-type Fus, R521G mutant and Fus $\Delta 32$ was confirmed to be consistent with the findings in cell culture, i.e. Fus $\Delta 32$ was largely diffused outside of the nucleus. Moreover, the fusion of an NES at the C-terminus of the full-length wild-type Fus dramatically reduced toxicity in vivo (Figures 5G, I and $6 \mathrm{~F}$ ). FusNES was also diffused in the motor neurons (Additional file 1: Figure S2), thus the data from both FusNES and Fus $\Delta 32$ suggest that the nuclear localization is critical to the toxicity. Furthermore, the chimeric protein Fus $\triangle 32$ NLS with an exogenous NLS was localized in the nucleus (Additional file 1: Figure S2) and produced similar toxicity as wild-type Fus (Figure $5 \mathrm{H}, \mathrm{I}$, and $6 \mathrm{G}$ ). All these results consistently suggest that the nuclear localization is required for Fus toxicity.

This surprising result differs from the common presumption that the toxicity is caused by the cytoplasmic accumulation of Fus. Our study is the first one characterizing transgenic flies expressing truncated Fus mutant with a primarily cytoplasmic localization. We further manipulated the subcellular localization by using a typical NES and the other different NLS. All experiments consistently support the conclusion. Interestingly, another study showed that mutation in the NLS of TDP-43 relieved the toxicity in C. elegans [36], supporting the conclusion of our study. However, the study by Lanson et al. employed another approach by deleting a postulated NES within the RNA recognition motif of
Fus and showed reduced toxicity [25]. It should be noted that Lanson et al. did not characterize the NES or the subcellular localization of the NES deletion mutant. Moreover, the postulated NES is within the RNA binding domain in Fus, therefore such deletion may cause a disruption of the RNA binding properties of Fus. Thus, the mechanism of the reduced toxicity of that particular NES deletion mutant needs to be better elucidated. We are confident in our conclusion since the results using three different approaches (Fus $\triangle 32$, FusNES, and Fus $\Delta 32$ NLS) consistently support the requirement of nuclear localization for Fus toxicity.

Another critical difference between this study and the study by Lanson et al. is how the transgenic flies were generated. The site-specific transgenic approach using the integrase-mediated insertion at the specific attP locus allowed us to ensure equal expression of the Fus proteins without positional effects. We think that the discrepancy is likely due to the expression level of the protein. This likely explains the discrepancy regarding the toxicity of the Fus transgene in different studies. The R521G mutation produced comparable levels of toxicity as wild-type Fus whereas the other study showed more severe toxicity in flies expressing $\mathrm{R} 521 \mathrm{H}$ or R521C [25]. The experiments in our study are more likely to produce comparable expression levels of the Fus transgene since transgene insertion was random in other studies.

Disruption of muscle 6/7 NMJs was observed in the Fus transgenic flies in this study. It is important to note that NMJ vulnerability occurs selectively in different subgroups of motor neurons or muscles. The diversity of subtypes of motor neurons and muscles and their susceptibility are well documented in ALS [41]. We examined the muscle 6/7 NMJs in the abdominal segment A2 of the third instar larvae, which is widely used in Drosophila studies. In addition, we used GFP as a marker to monitor the target protein expression. Our results showed the expression of Fus/Caz in the disrupted NMJs. Studies characterizing NMJs in other muscles may obtain different results, for example another study examined muscle 4, segment A2 and 3 and did not observe reduction of synaptic boutons in Fus overexpressing flies [25].

Notably, neither wild-type Fus/Caz nor the ALS mutants of Fus showed cytoplasmic inclusions in transgenic flies where severe toxicity was demonstrated in this study. The results suggest that the cytoplasmic inclusions are not essential to Fus toxicity although they have been prominently observed in cell culture systems and in human patient tissues. It has long been proposed that soluble oligomers may be the culprit species causing neurodegenerative disease. A recent study showed that aberrant high molecular weight complex of TDP-43 was toxic, although 
the nature of the species was still unknown [42]. A recent study also showed that Fus toxicity could be suppressed without eliminating protein inclusions in yeast [40], suggesting that protein inclusions might be a non-toxic and non-essential feature of the disease.

Although the results in this study consistently suggest that sufficient steady-state levels of Fus/Caz are required for the toxicity and neurodegenerative phenotypes, it is noted that Fus/Caz undergoes dynamic trafficking in and out of the nucleus in live cells. It remains possible that Fus/Caz with steady-state nuclear localization could produce detrimental effect while it is temporarily outside of the nucleus. Alternatively, the minute amount of Fus/Caz in the cytoplasm that cannot be detected by confocal microscope could also produce cytosolic toxicity. We believe that these two are remote possibilities, however additional studies using more sophisticated approaches are needed to test them in the future. Moreover, the exact nature of Fus toxicity in the nucleus also remains to be determined in future studies.

\section{The phenotypes of caz deletion in Drosophila}

Deletion of a specific gene provides insight into understanding the in vivo function of the protein. By taking advantage of the Drosophila model, we found that endogenous Caz is required for normal functions of the neurons (Figure 7). The deficiency of caz in flies induced a strong eye phenotype with disrupted ommatidia structure (Figure 7A-I) and caused defects in the locomotive function in larvae (Figure 7K) and adult flies (Figure 7J). Consistently, caz1 mutant flies exhibited decreased adult viability and diminished locomotive activity [27]. These phenotypes were actually similar to those caused by Fus/Caz overexpression. It would be interesting to examine whether some cases of ALS disease may be caused by Fus loss-of-function in patients.

Given the motor deficiencies of Fus overexpression and mutant caz flies, the presynaptic structure in NMJ was examined. As expected, the number of boutons was significantly reduced in the larvae overexpressing Fus (Figure 4) as well as in the larvae lacking caz (Figure 7L-M). Thus the disruption of NMJ was evidently the cause of the locomotive defects. Therefore, the Fus/Caz overexpression and loss of caz obviously caused similar disruption of presynaptic terminals in NMJs. It is likely that $\mathrm{Caz}$ is required for neuronal function and therefore the lack of Fus/Caz perturbs the motor neuron distal terminus and causes NMJ disruption.

\section{Different molecular mechanisms for degenerative phenotypes in Fus overexpression and caz deletion Drosophila}

By examining the nuclear structure and monitoring apoptosis of cells in the VNC, we found that overexpression of
Fus/Caz induced apoptotic cell death (Figure 6). However, neither the caz deficiency (BSC759) nor the caz1 mutation led to apoptosis (Figure 7P and 7S, data not shown). Despite the critical difference, both Fus/Caz overexpression and caz deletion disrupted the NMJs. This raises the question of where the toxicity originates, either from the death of neuronal cell bodies or disruption of the NMJs.

Studies in the SOD1 mutant-mediated familial ALS have suggested an axon die-back model in which perturbation at the distal NMJs occurs prior to the death of motor neurons [41]. The Fus/Caz overexpression phenotypes support this model, but the time sequence of the NMJ disruption and motor neuron death has not been distinguished in these flies as yet. Moreover, both the caz deficiency and caz1 mutant flies strongly support this model since loss of Fus/Caz caused NMJ disruption and motor function deficiency without the presence of motor neuronal cell death. The findings in our study support two potential mechanisms for the Fus/Caz mediated neurotoxicity: neuronal apoptosis and NMJ perturbation. These two mechanisms are not necessarily mutually exclusive, and in fact may occur simultaneously. Further studies exploring of the in-depth mechanisms by which Fus/Caz causes alterations in RNA processing and nuclear toxicity are currently ongoing. Many laboratories in the field also use various models, including yeast $[39,40,43,44]$ and Drosophila [25-27], to investigate the role of Fus in RNA metabolism and the genetic interactions between Fus and other RNA processing proteins. The overexpression and deletion Drosophila models in this study provide valuable insight into the etiology of ALS and potentially other degenerative diseases in which RNA processing proteins are implicated.

\section{Conclusions}

We conclude from our study that the expression of Fus can cause nuclear toxicity, induce apoptosis and facilitate NMJ perturbation in the transgenic Drosophila model of ALS. Generation and characterization of the Drosophila model of Fus-mediated ALS have revealed several novel findings and provided new insights into the disease etiology. There are three major novel findings in this study: (1) the C-terminus of Fus is essential for its in vivo toxicity, suggesting that Fus toxicity originates from the nuclei instead of the cytoplasm; (2) both the overexpression of Fus/Caz and the deletion of $\mathrm{caz}$ caused similar deficiency in locomotive function and disruption in NMJs; (3) the overexpression of Fus/Caz induced apoptosis in motor neuron cell bodies, whereas no apoptosis was observed in the motor neurons of the caz mutant flies. In addition to the novel findings, we have also developed assays to investigate the functions of Fus/Caz in the Drosophila VNC. The novel neuronal 
functions of Caz that we have uncovered here are likely to be conserved in other species.

\section{Methods}

Constructs, mutants, and transgenes

UAST-Myc-Fus was generated by subcloning the cDNA fragment of human Fus into the attB-UAST-5xMyc vector. Amino acid substitutions of R521G, R521C, and Y526F were generated by PCR-based site-directed mutagenesis. Fus $\Delta 32$ and Fus1-453 were generated by truncation at F494 and G453, respectively. FusR521G, FusR521C, FusY526F, Fus1-453, and Fus $\Delta 32$ were subcloned into attB-UAST-5xMyc using the same approach as used for Fus. To generate FusNES, the typical NES sequence (NINELALKFAGLDI) [45] was fused in frame with the C-terminus of Myc-Fus. To generate Fus $\triangle 32$ NLS, the NLS sequence (YGDYSNQQSGYGKVSRRGGHQNSYKPY) from hnRNP D [46] was fused in frame with Fus $\Delta 32$. To generate HA-tagged Caz (HA-Caz), we obtained cDNA clone (LD22761) from the Drosophila Genome Resource Center (DGRC, USA) and subcloned the full-length Caz cDNA into attB-UAST-HA vector. To ensure that the proteins were expressed at the same level without a positional effect, the PhiC31 integration system was used to integrate Fus and Caz transgenes into the 75B1 attP locus in the fly genome $[30,31]$. The Caz RNAi line, v100291, and the UAS-Dicer lines were obtained from the VDRC (Austria). $D F(1) B S C 759$ and $D F(1) E D 7355$ were obtained from the Bloomington Drosophila Stock Center (USA). caz1 mutant flies carry deletion Df[1]383 and a genomic transgene for CG32576, which is supposed to be a caz null [27]. MS1096-Gal4; D42-Gal4, Elav-Gal4, act5CGal4, GMR-Gal4, OK371-Gal4, UAS-Dicer, and $y w$ have been previously described (Flybase) [31].

\section{Adult eye histology, immunoblot analysis, and eye imaginal disc immunostaining}

To analyze the internal structure of adult eyes, the heads of flies from specific genotypes at 4 day-after-eclosion (DAE) were fixed in Bouin's fixative solution (Cat\# HT10132, Sigma, USA) for $48 \mathrm{~h}$ at room temperature on a shaker at low speed, incubated $24 \mathrm{~h}$ in $50 \mathrm{mM}$ Tris/150 $\mathrm{mM} \mathrm{NaCl}$, and then embedded in paraffin. Serial sections (5 $\mu \mathrm{m}$ thickness) were taken through the entire head, stained with Eosin (Cat\# S176, Poly Scientific, USA), and examined under a Leica DM750 microscope (Leica, Germany). Western blot was performed to confirm comparable levels of protein expression. Fifteen heads from adults expressing different Fus or Caz transgenes by GMR-Gal4 were dissected and subjected to direct Western blot with mouse anti-Myc (9E10, 1:5000, Santa Cruz, USA) and anti- $\beta$-tubulin (1:5000, Developmental Studies Hybridoma Bank; DSHB, USA). A standard protocol was used for the eye imaginal disc immunostaining assay. Eye discs were dissected, fixed in $4 \%$ paraformaldehyde, washed in phosphate-buffered saline with $0.3 \%$ Triton-X100 (PBT), and then stained with mouse anti-Elav (1:50, DSHB) for $2 \mathrm{~h}$ at room temperature. After washing with PBT, the eye discs were stained with goat anti-mouse Rhodamin (1:500, Jackson ImmunoResearch, USA) and rabbit anti-Horseradish Peroxidase (HRP) conjugated to Cy5 (1:500, Jackson ImmunoResearch) for 2 hours at room temperature.

\section{Dissection, immunostaining, and analysis of NMJs from third instar larvae}

Late third instar larvae were dissected, fixed in $4 \%$ paraformaldehyde, washed in PBT, and stained with an antiDiscs-large (Dlg) antibody (1:100, DSHB) at room temperature for $2 \mathrm{~h}$. After washing with PBT, the discs were stained with goat anti-mouse Rhodamin (1:500, Jackson ImmunoResearch) and rabbit anti-HRP antibody conjugated to Cy5 (Jackson ImmunoResearch, 1:500). Samples were then mounted and viewed with an Olympus confocal microscope. Z-scan sections of the NMJs from ventral longitudinal muscles 6 and 7 at segment A2 were taken at $1.0 \mu \mathrm{m}$ intervals by confocal imaging (Olympus Fluoview, Ver.1.7c) to visualize the HRP-positive neuronal membrane and the Dlg-positive large boutons. The number of large and small boutons was also quantified after intensity projection over the $\mathrm{Z}$ axis.

\section{Immunostaining and TUNEL assay of the VNCs from third} instar larvae

Fly larval VNCs with brains from specific genotypes were dissected, fixed in $4 \%$ paraformaldehyde, and immunostained with mouse anti-Myc (9E10, 1:50, Santa Cruz) or anti-HA (F-7, 1:150, Santa Cruz) for $2 \mathrm{~h}$ at room temperature. After washing with PBT, the tissues were stained with goat anti-mouse Rhodamin (1:500, Jackson ImmunoResearch). Hoechst dye $33342(1 \mu \mathrm{g} / \mathrm{ml}$, Invitrogen, USA) was added with the secondary antibody staining. For TUNEL staining, samples were pretreated with $3 \mu \mathrm{g} / \mathrm{ml}$ Proteinase $\mathrm{K}$ for $10 \mathrm{~min}$ at room temperature, fixed in $4 \%$ paraformaldehyde, and permeabilized in PBT with $0.1 \%$ sodium citrate. Samples were then treated with TUNEL (In Situ Cell Death Detection Kit) with green fluorescence (Roche, Cat\# 11684795910) followed by imaging analysis with the Olympus confocal microscope.

Western blot was performed to confirm protein expression at a comparable level. Five brains containing VNCs from larvae expressing Fus variants or Caz by OK371-Gal4 were dissected and lysed in regular Schneider $\mathrm{S} 2$ cell lysis buffer, and then subjected to direct Western blot with mouse anti-Myc (9E10, 1:5000, Santa Cruz), anti-HA (F-7, 1:1500, Santa Cruz), or anti-GFP (1:1000, Millipore, USA). 


\section{Larval movement and adult climbing assays}

Third instar larvae bearing specific genotypes were subjected to motor function analysis on $100 \mathrm{~mm}$ agar plates. Individual larvae were placed on plates and stimulated to move with a needle. The distance they traveled over the next $30 \mathrm{~s}$ was traced and measured. Each individual larva was counted 3 times and 20 larvae were tested for each group. The climbing ability of adult flies with different genotypes was examined in empty $2 \mathrm{ml}$ pipettes vertically. Flies were first placed at the bottom of the pipette and the climbing distances were scored over $30 \mathrm{~s}$. Each fly was recorded 3 times and 20 flies in each group were tested.

\section{Additional material}

Additional file 1: Figure S1 The caz gene encodes the Drosophila homolog of Fus. A sequence alignment between Caz, mouse Fus, and human Fus is shown here. The SYQG domain, G-rich domain, RNA binding domain, Zn-finger domain, and RGG-rich domains are underlined. The asterisks indicate the ALS-related mutations that occur in patients. Additional file 1: Figure S2 Large magnification of the motor neuronal cells indicating the localization of the proteins expressed in VNCs from Figure 5. Fus, Caz, and Fus $\triangle 32$ NLS are exclusively localized in the nucleus. FusR521G shows slightly increased staining in the cytosol, but Fus $\triangle 32$ and FusNES are mainly localized in the cytoplasm. Additional file 1: Figure S3 Mutating caz in flies causes severe defects in the eye. (A) A female $y w$ fly showing adult wild-type morphology. (B) A caz heterozygous female fly shows the balancer marker and intact eye structure. (C) A caz homozygous female fly shows growth defects in the eye and exhibits a normal body. (D) A male yw fly showing adult wildtype morphology. (E) A caz mutant male fly shows growth defects in the eye and exhibits a normal body. Mutating caz causes severely disrupted locomotive ability (Figure 7) without obvious changes in body size, suggesting that $\mathrm{Caz}$ is likely involved in neurodegeneration. Additional file 1: Table S1 Characterization of Fus toxicity in Drosophila. Shown here is a diagram illustrating the domain structure of Fus along with truncation and point mutation constructs that are expressed in flies by the indicated Gal4 lines. The levels of toxicity are determined in the wing (by MS1096-Gal4), the eye (by GMRGal4), the entire body (by the act5CGal4), and the neuron (by Elav-Gal4 and D42-Gal4). More "+" symbols indicates more severe phenotypes.

\section{Abbreviations}

ALS: Amyotrophic lateral sclerosis; Fus: Fused in sarcoma; Caz: Cabeza; VNC: Ventral nerve cord; NLS: Nuclear localization sequence; NES: Nuclear export signal; SOD1: Cu/Zn superoxide dismutase; NMJ: Neuromuscular junction.

\section{Acknowledgements}

We thank Mary K. Brewer for technical assistance, and Drs. Hongge Jia and Jiayu Zhang for making some of the Fus/Caz constructs. We thank the Bonini Lab for providing a detailed protocol of the fly eye paraffin section. We thank Dr. Brian McCabe for the cazl mutant. We thank the Developmental Studies Hybridoma Bank (DSHB) for antibodies and the Bloomington Stock Center for Drosophila strains. This work was in part supported by the National Institutes of Health grants (R01NS077284, R01NS049126 and R21AG032567 to HZ), the ALS Association (6SE340 to HZ), and the Center of Biomedical Research Excellence in the Molecular Basis of Human Disease (COBRE, P2ORR020171).

\section{Author details}

'Markey Cancer Center, University of Kentucky, KY 40536 Lexington, USA. ${ }^{2}$ Department of Molecular and Cellular Biochemistry, College of Medicine, University of Kentucky, KY 40536 Lexington, USA.

\section{Authors' contributions}

$R X, Y L, L Y, J G$ performed the experiments. $H Z$ and JJ analyzed the results, designed the study, and wrote the manuscript. All authors read and approved the final manuscript.

\section{Competing interests}

The authors declare that they have no competing interests.

Received: 20 January 2012 Accepted: 24 March 2012 Published: 24 March 2012

\section{References}

1. Boillee S, Vande Velde C, Cleveland DW: ALS: a disease of motor neurons and their nonneuronal neighbors. Neuron 2006, 52(1):39-59.

2. Valdmanis PN, Rouleau GA: Genetics of familial amyotrophic lateral sclerosis. Neurology 2008, 70(2):144-152.

3. Rosen DR, Siddique T, Patterson D, Figlewicz DA, Sapp P, Hentati A Donaldson D, Goto J, O'Regan JP, Deng HX, et al: Mutations in Cu/Zn superoxide dismutase gene are associated with familial amyotrophic lateral sclerosis. Nature 1993, 362(6415):59-62.

4. Sreedharan J, Blair IP, Tripathi VB, Hu X, Vance C, Rogelj B, Ackerley S, Durnall JC, Williams KL, Buratti E, et al: TDP-43 mutations in familial and sporadic amyotrophic lateral sclerosis. Science 2008, 319(5870):1668-1672.

5. Sapp PC, Hosler BA, McKenna-Yasek D, Chin W, Gann A, Genise H, Gorenstein J, Huang M, Sailer W, Scheffler M, et al: Identification of two novel loci for dominantly inherited familial amyotrophic lateral sclerosis. Am J Hum Genet 2003, 73(2):397-403.

6. Ruddy DM, Parton MJ, Al-Chalabi A, Lewis CM, Vance C, Smith BN, Leigh PN, Powell JF, Siddique T, Meyjes EP, et al: Two families with familial amyotrophic lateral sclerosis are linked to a novel locus on chromosome 16q. Am J Hum Genet 2003, 73(2):390-396.

7. Kwiatkowski TJ, Bosco DA, LeClerc AL, Tamrazian E, Vanderburg CR, Russ C, Davis A, Gilchrist J, Kasarskis EJ, Munsat T, et al: Mutations in the FUS/TLS gene on chromosome 16 cause familial amyotrophic lateral sclerosis. Science 2009, 323(5918):1205-1208.

8. Vance C, Rogelj B, Hortobagyi T, De Vos K, Nishimura AL, Sreedharan J, Hu X, Smith B, Ruddy D, Wright P, et al: Mutations in FUS, an RNA processing protein, cause familial amyotrophic lateral sclerosis type 6 . Science 2009, 323(5918):1208-1211.

9. Belzil W, Valdmanis PN, Dion PA, Daoud H, Kabashi E, Noreau A, Gauthier J, Hince P, Desjarlais A, Bouchard JP, et al: Mutations in FUS cause FALS and SALS in French and French Canadian populations. Neurology 2009, 73(15):1176-1179.

10. Corrado L, Del Bo R, Castellotti B, Ratti A, Cereda C, Penco S, Soraru G, Carlomagno Y, Ghezzi S, Pensato V, et al: Mutations of FUS gene in sporadic amyotrophic lateral sclerosis. J Med Genet 2010, 47(3):190-194.

11. DeJesus-Hernandez M, Kocerha J, Finch N, Crook R, Baker M, Desaro P, Johnston A, Rutherford N, Wojtas A, Kennelly K, et al: De novo truncating FUS gene mutation as a cause of sporadic amyotrophic lateral sclerosis. Hum Mutat 2010, 31(5):E1377-E1389.

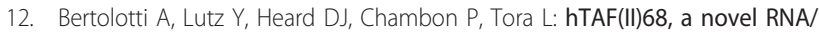
ssDNA-binding protein with homology to the pro-oncoproteins TLS/FUS and EWS is associated with both TFIID and RNA polymerase II. EMBO J 1996, 15(18):5022-5031.

13. Zinszner $H$, Sok J, Immanuel D, Yin $Y$, Ron D: TLS (FUS) binds RNA in vivo and engages in nucleo-cytoplasmic shuttling. J Cell Sci 1997, 110(Pt 15): $1741-1750$

14. Lagier-Tourenne C, Polymenidou M, Cleveland DW: TDP-43 and FUS/TLS: emerging roles in RNA processing and neurodegeneration. Hum Mol Genet 2010, 19(R1):R46-R64.

15. Fujii R, Okabe $\mathrm{S}$, Urushido T, Inoue $K$, Yoshimura A, Tachibana T, Nishikawa T, Hicks GG, Takumi T: The RNA binding protein TLS is translocated to dendritic spines by mGluR5 activation and regulates spine morphology. Curr Biol 2005, 15(6):587-593.

16. Fujii $R$, Takumi T: TLS facilitates transport of mRNA encoding an actinstabilizing protein to dendritic spines. J Cell Sci 2005, 118(Pt 24):5755-5765.

17. Andersson MK, Stahlberg A, Arvidsson Y, Olofsson A, Semb H, Stenman G, Nilsson O, Aman P: The multifunctional FUS, EWS and TAF15 protooncoproteins show cell type-specific expression patterns and 
involvement in cell spreading and stress response. BMC Cell Biol 2008, 9:37.

18. Aman P, Panagopoulos I, Lassen C, Fioretos T, Mencinger M, Toresson $H_{\text {, }}$ Hoglund M, Forster A, Rabbitts TH, Ron D, et al: Expression patterns of the human sarcoma-associated genes FUS and EWS and the genomic structure of FUS. Genomics 1996, 37(1):1-8.

19. Gal J, Zhang J, Kwinter DM, Zhai J, Jia H, Jia J, Zhu H: Nuclear localization sequence of FUS and induction of stress granules by ALS mutants. Neurobiol Aging 2011, 32(12):2323, e27-40.

20. Dormann D, Rodde R, Edbauer D, Bentmann E, Fischer I, Hruscha A, Than ME, Mackenzie IR, Capell A, Schmid B, et al: ALS-associated fused in sarcoma (FUS) mutations disrupt Transportin-mediated nuclear import. EMBO J 2010, 29(16):2841-2857.

21. Bosco DA, Lemay N, Ko HK, Zhou H, Burke C, Kwiatkowski TJ Jr, Sapp P, McKenna-Yasek D, Brown RH Jr, Hayward LJ: Mutant FUS proteins that cause amyotrophic lateral sclerosis incorporate into stress granules. Hum Mol Genet 2010, 19(21):4160-4175.

22. Feiguin F, Godena VK, Romano G, D'Ambrogio A, Klima R, Baralle FE: Depletion of TDP-43 affects Drosophila motoneurons terminal synapsis and locomotive behavior. FEBS Lett 2009, 583(10):1586-1592.

23. Li Y, Ray P, Rao EJ, Shi C, Guo W, Chen X, Woodruff EA, Fushimi K, Wu JY: A Drosophila model for TDP-43 proteinopathy. Proc Nat Acad Sci USA 2010, 107(7):3169-3174.

24. Ritson GP, Custer SK, Freibaum BD, Guinto JB, Geffel D, Moore J, Tang W, Winton MJ, Neumann M, Trojanowski JQ, et al: TDP-43 mediates degeneration in a novel Drosophila model of disease caused by mutations in VCP/p97. J Neurosci 2010, 30(22):7729-7739.

25. Lanson NA Jr, Maltare A, King H, Smith R, Kim JH, Taylor JP, Lloyd TE, Pandey UB: A Drosophila model of FUS-related neurodegeneration reveals genetic interaction between FUS and TDP-43. Hum Mol Genet 2011, 20(13):2510-2523.

26. Chen Y, Yang M, Deng J, Chen X, Ye Y, Zhu L, Liu J, Ye H, Shen Y, Li Y, et al: Expression of human FUS protein in Drosophila leads to progressive neurodegeneration. Protein Cell 2011, 2(6):477-486.

27. Wang JW, Brent JR, Tomlinson A, Shneider NA, McCabe BD: The ALSassociated proteins FUS and TDP-43 function together to affect Drosophila locomotion and life span. J Clin Invest 2011, 121(10):4118-26.

28. Immanuel D, Zinszner H, Ron D: Association of SARFH (sarcomaassociated RNA-binding fly homolog) with regions of chromatin transcribed by RNA polymerase II. Mol Cell Biol 1995, 15(8):4562-4571.

29. Stolow DT, Haynes SR: Cabeza, a Drosophila gene encoding a novel RNA binding protein, shares homology with EWS and TLS, two genes involved in human sarcoma formation. Nucleic Acids Res 1995, 23(5):835-843.

30. Bischof J, Maeda RK, Hediger M, Karch F, Basler K: An optimized transgenesis system for Drosophila using germ-line-specific phiC31 integrases. Proc Nat Acad Sci USA 2007, 104(9):3312-3317.

31. Jia H, Liu Y, Xia R, Tong C, Yue T, Jiang J, Jia J: Casein kinase 2 promotes Hedgehog signaling by regulating both smoothened and Cubitus interruptus. J Biol Chem 2010, 285(48):37218-37226.

32. Bilen J, Bonini NM: Drosophila as a model for human neurodegenerative disease. Annu Rev Genet 2005, 39:153-171.

33. Johnson BS, MCCaffery JM, Lindquist S, Gitler AD: A yeast TDP-43 proteinopathy model: Exploring the molecular determinants of TDP-43 aggregation and cellular toxicity. Proc Nat Acad Sci USA 2008, 105(17):6439-6444.

34. Hanson KA, Kim SH, Wassarman DA, Tibbetts RS: Ubiquilin modifies TDP43 toxicity in a Drosophila model of amyotrophic lateral sclerosis (ALS). $J$ Biol Chem 2010, 285(15):11068-11072.

35. Elden AC, Kim HJ, Hart MP, Chen-Plotkin AS, Johnson BS, Fang X, Armakola M, Geser F, Greene R, Lu MM, et al: Ataxin-2 intermediate-length polyglutamine expansions are associated with increased risk for ALS. Nature 2010, 466(7310):1069-1075.

36. Ash PE, Zhang YJ, Roberts CM, Saldi T, Hutter H, Buratti E, Petrucelli L, Link CD: Neurotoxic effects of TDP-43 overexpression in C. elegans. Hum Mol Genet 2010, 19(16):3206-3218

37. Wegorzewska I, Bell S, Cairns NJ, Miller TM, Baloh RH: TDP-43 mutant transgenic mice develop features of ALS and frontotemporal lobar degeneration. Proc Natl Acad Sci 2009, 106(44):18809-18814.

38. Igaz LM, Kwong LK, Lee EB, Chen-Plotkin A, Swanson E, Unger T, Malunda J, Xu Y, Winton MJ, Trojanowski JQ, Lee VMY: Dysregulation of the ALS-associated gene TDP-43 leads to neuronal death and degeneration in mice. $J$ Clin Invest 2011, 121(2):726-738.

39. Sun Z, Diaz Z, Fang X, Hart MP, Chesi A, Shorter J, Gitler AD: Molecular Determinants and Genetic Modifiers of Aggregation and Toxicity for the ALS Disease Protein FUS/TLS. PLOS Biol 2011, 9(4):e1000614.

40. Ju S, Tardiff DF, Han H, Divya K, Zhong Q, Maquat LE, Bosco DA, Hayward LJ, Brown RH Jr, Lindquist S, et al: A Yeast Model of FUS/TLSDependent Cytotoxicity. PLOS Biol 2011, 9(4):e1001052

41. Kanning KC, Kaplan A, Henderson CE: Motor neuron diversity in development and disease. Annu Rev Neurosci 2010, 33:409-440.

42. Guo W, Chen $Y$, Zhou X, Kar A, Ray P, Chen X, Rao EJ, Yang M, Ye H, Zhu L, et al: An ALS-associated mutation affecting TDP-43 enhances protein aggregation, fibril formation and neurotoxicity. Nat Struct Mol Biol 2011, 18(7):822-830.

43. Fushimi K, Long C, Jayaram N, Chen X, Li L, Wu JY: Expression of human FUS/TLS in yeast leads to protein aggregation and cytotoxicity, recapitulating key features of FUS proteinopathy. Protein Cell 2011, 2(2):141-149.

44. Kryndushkin D, Wickner RB, Shewmaker F: FUS/TLS forms cytoplasmic aggregates, inhibits cell growth and interacts with TDP-43 in a yeast model of amyotrophic lateral sclerosis. Protein Cell 2011, 2(3):223-236.

45. Guttler T, Madl T, Neumann P, Deichsel D, Corsini L, Monecke T, Ficner R, Sattler M, Gorlich D: NES consensus redefined by structures of PKI-type and Rev-type nuclear export signals bound to CRM1. Nat Struct Mol BiOl 2010, 17(11):1367-1376.

46. Lee BJ, Cansizoglu AE, Suel KE, Louis TH, Zhang Z, Chook YM: Rules for nuclear localization sequence recognition by karyopherin beta 2 . Cell 2006, 126(3):543-558

doi:10.1186/1750-1326-7-10

Cite this article as: Xia et al:: Motor neuron apoptosis and neuromuscular junction perturbation are prominent features in a Drosophila model of Fus-mediated ALS. Molecular Neurodegeneration 2012 7:10.

\section{Submit your next manuscript to BioMed Central and take full advantage of:}

- Convenient online submission

- Thorough peer review

- No space constraints or color figure charges

- Immediate publication on acceptance

- Inclusion in PubMed, CAS, Scopus and Google Scholar

- Research which is freely available for redistribution

Submit your manuscript at www.biomedcentral.com/submit
C Biomed Central 